\title{
The Bacterial Effector AvrPto Targets the Regulatory Coreceptor SOBIR1 and Suppresses Defense Signaling Mediated by the Receptor-Like Protein Cf-4
}

\author{
Jinbin Wu, ${ }^{1}$ Aranka M. van der Burgh, ${ }^{1}$ Guozhi Bi, ${ }^{1}$ Lisha Zhang, ${ }^{1}$ James R. Alfano, ${ }^{2,3}$ \\ Gregory B. Martin, ${ }^{4,5}$ and Matthieu H. A. J. Joosten ${ }^{1,+}$ \\ ${ }^{1}$ Laboratory of Phytopathology, Wageningen University, Droevendaalsesteeg 1, 6708 PB Wageningen, The Netherlands; \\ ${ }^{2}$ Center for Plant Science Innovation and ${ }^{3}$ Department of Plant Pathology, University of Nebraska, Lincoln, NE 68588, U.S.A.; \\ ${ }^{4}$ Boyce Thompson Institute for Plant Research, Ithaca, NY 14853, U.S.A.; and ${ }^{5}$ Section of Plant Pathology and Plant-Microbe \\ Biology, School of Integrative Plant Science, Cornell University, Ithaca, NY 14853, U.S.A.
}

Accepted 31 August 2017.

Receptor-like proteins (RLPs) and receptor-like kinases (RLKs) are cell-surface receptors that are essential for detecting invading pathogens and subsequent activation of plant defense responses. RLPs lack a cytoplasmic kinase domain to trigger downstream signaling leading to host resistance. The RLK SOBIR1 constitutively interacts with the tomato RLP Cf-4, thereby providing $\mathrm{Cf}-4$ with a kinase domain. SOBIR1 is required for $\mathrm{Cf}-4-$ mediated resistance to strains of the fungal tomato pathogen Cladosporium fulvum that secrete the effector Avr4. Upon perception of this effector by the Cf-4/SOBIR1 complex, the central regulatory RLK SOMATIC EMBRYOGENESIS RECEPTOR KINASE 3a (SERK3a) is recruited to the complex and defense signaling is triggered. SOBIR1 is also required for RLP-mediated resistance to bacterial, fungal ,and oomycete pathogens, and we hypothesized that SOBIR1 is targeted by effectors of such pathogens to suppress host defense responses. In this study, we show that Pseudomonas syringae pv. tomato DC3000 effector AvrPto interacts with Arabidopsis SOBIR1 and its orthologs of tomato and Nicotiana benthamiana, independent of SOBIR1 kinase activity. Interestingly, AvrPto suppresses Arabidopsis SOBIR1-induced cell death in N. benthamiana. Furthermore, AvrPto compromises Avr4-triggered cell death in $C f$-4-transgenic $N$. benthamiana, without affecting Cf-4/SOBIR1/SERK3a complex formation. Our study shows that the RLP coreceptor SOBIR1 is targeted by a bacterial effector, which results in compromised defense responses.

The innate immune system of plants against invading pathogens consists of two layers, which are termed microbeassociated molecular pattern (MAMP)-triggered immunity

Current address for Guozhi Bi: The State Key Laboratory of Plant Genomics, Institute of Genetics and Developmental Biology, Chinese Academy of Sciences, Beijing 100101, China

Current address for Lisha Zhang: Center of Plant Molecular Biology (ZMBP), University of Tübingen, Auf der Morgenstelle 32, Tübingen D-72076, Germany

${ }^{\dagger}$ Corresponding author: Matthieu H. A. J. Joosten;

E-mail: Matthieu.Joosten@wur.nl

*The $\boldsymbol{e}$-Xtra logo stands for "electronic extra" and indicates that one supplementary figure is published online.

() 2018 The American Phytopathological Society
(MTI) and effector-triggered immunity (ETI) (Couto and Zipfel 2016; Janeway 1989; Jones and Dangl 2006; Macho and Zipfel 2015). MAMPs are conserved structural components of pathogens, whereas effectors are typically in planta-induced proteins of microbial pathogens. Effectors suppress plant defense responses, thereby causing effector-triggered susceptibility (ETS) (Dodds and Rathjen 2010; Feng and Zhou 2012; Jones and Dangl 2006). Essentially, there are two subcellular locations of pathogen perception, the apoplast and the cytoplasm (Cui et al. 2009; Dodds and Rathjen 2010; Zipfel 2014). MAMPs and secreted effectors that end up in the apoplast are generally recognized by plasma membrane-associated pattern recognition receptors (PRRs) (Dodds and Rathjen 2010; Zipfel 2014). Effectors that are translocated from fungal or oomycete haustoria, which are specialized feeding structures that are formed in host cells, or are injected into the cell by the type three secretion system (T3SS) of bacteria are perceived by cytoplasmic immune receptors (Dodds and Rathjen 2010). These cytoplasmic receptors mostly carry a nucleotide binding site and leucine-rich repeats (NB-LRRs) (Dodds and Rathjen 2010).

PRRs are either receptor-like kinases (RLKs) or receptor-like proteins (RLPs) (Couto and Zipfel 2016; Zipfel 2014). Compared with RLKs, RLPs lack a kinase domain to trigger downstream signaling. Recently it was observed that RLPs constitutively interact with the RLK SUPPRESSOR OF BAK1-INTERACTING RECEPTOR-LIKE KINASE 1 (SOBIR1) and require SOBIR1 for their function (Gust and Felix 2014; Liebrand et al. 2013, 2014). Arabidopsis thaliana SOBIR1 was originally identified as a suppressor of the birl-1 phenotype, partially rescuing birl knockout plants that mount a constitutive defense response (Gao et al. 2009). AtSOBIR1 was found to function as a positive regulator of cell death, as overexpression of AtSOBIRl triggered enhanced basal defense and reduced colonization by the bacterial pathogen Pseudomonas syringae pv. tomato DC3000 (Gao et al. 2009). Recently, the RLK SOMATIC EMBRYOGENESIS RECEPTOR KINASE 3 (SERK3)/BRASSINOSTEROID INSENSITIVE 1 (BRI1)-ASSOCIATED RECEPTOR KINASE 1 (BAK1), further referred to as SERK3, has been reported as another suppressor of birl-1 (Liu et al. 2016). Both SOBIR1 and SERK3 are required for birl-1-triggered autoimmunity, and interaction was found between SOBIR1 and SERK3 in BIR1-silenced Nicotiana benthamiana (Liu et al. 2016).

The tomato (Solanum lycopersicum) RLP Cf-4 confers resistance to the pathogenic biotrophic fungus Cladosporium fulvum, by recognition of the secreted effector Avr4. It was 
recently shown that SERK3 is also required for Cf-4 signaling and is recruited to the Cf-4/SOBIR1 complex upon recognition of Avr4 (Postma et al. 2016). Increasing evidence on signaling by RLP/SOBIR1/SERK3-containing complexes indicates that SOBIR1 constitutively forms a complex with RLPs, whereas SERK3 is specifically recruited to the RLP/SOBIR1 bipartite RLK upon ligand recognition by the RLP (Albert et al. 2015; Zhang et al. 2013a). SOBIR1 and SERK3 are also required for tomato Ve1-, I-, and Brassica napus LepR3-mediated resistance to Verticillium dahliae expressing Ave1 (Liebrand et al. 2013), Fusarium oxysporum f. sp. lycopersici expressing Avr1 (Catanzariti et al. 2017), and Leptosphaeria maculans expressing AvrLm1 (Ma and Borhan 2015), respectively, although for these particular RLPs the association of SERK3 with the RLP/SOBIR1 complex remains to be shown. SOBIR1 is also required for the functionality of various additional RLPs playing a role in immunity (Hegenauer et al. 2016; Jehle et al. 2013; Zhang et al. 2014), though the requirement of SERK3 for the functionality of these RLPs remains currently unknown. Furthermore, SOBIR1 is involved in resistance to the oomycete pathogen Phytophthora parasitica (Peng et al. 2015) and the fungus Magnaporthe oryzae (Takahashi et al. 2016).

The Arabidopsis RLK FLAGELLIN-SENSING 2 (FLS2) confers resistance to $P$. syringae pv. tomato DC3000 (Couto and Zipfel 2016). Flg22, a 22-amino acid peptide derived from a conserved domain of bacterial flagellin, is perceived by FLS2 (Gómez-Gómez and Boller 2000) together with SERK3 (Chinchilla et al. 2007; Sun et al. 2013). Recognition of flg22 triggers a rapid defense response, including calcium spiking (Boudsocq et al. 2010), reactive oxygen species synthesis (Kadota et al. 2015; Ma 2014) and MITOGENACTIVATED PROTEIN KINASE (MAPK) activation (Asai et al. 2002). The receptor-like cytoplasmic kinase (RLCK) BOTRYTISINDUCED KINASE1 (BIK1) constitutively interacts with FLS2 and SERK3, and association with FLS2 or SERK3 is not affected in single serk3- or fls2-knockout mutants, respectively (Lu et al. 2010). However, flg22-induced BIK1 phosphorylation is both FLS2- and SERK3-dependent, as BIK1 phosphorylation does not take place in fls2 or serk3 mutants (Lu et al. 2010). BIK1 is a substrate of SERK3, which, in turn, phosphorylates SERK3 and FLS2 (Lu et al. 2010). After flg22 perception, BIK1 is released from FLS2 and triggers early defense responses (Ma 2014; Zhang et al. 2010).

Pathogenic bacteria inject their effectors into the host cells via the T3SS to suppress MTI by directly interfering with PRR function or with downstream signaling pathways (Bi and Zhou 2017; Couto and Zipfel 2016; Lee et al. 2013; Tang et al. 2017). For example, the effector AvrPto, also referred to as AvrPto1, is injected into host cells by $P$. syringae pv. tomato DC3000 and localizes to the plasma membrane (Shan et al. 2000). AvrPto targets FLS2 and suppresses the phosphorylation of its kinase domain (Xiang et al. 2008). AvrPto suppresses the flg22activated MTI (He et al. 2006), although it is not clear if SERK3 is also targeted by AvrPto (Shan et al. 2008; Xiang et al. 2008, 2011). Eventually, also BIK1 phosphorylation is suppressed, and the MAPK signaling pathway is inhibited (He et al. 2006; Lu et al. 2010; Xiang et al. 2011).

The resistance gene $P$ to (for resistance against $P$. syringae pv. tomato race 0) encodes a protein kinase with serine-threonine specificity (Loh and Martin 1995), which competes with FLS2 for AvrPto to trigger ETI (Xiang et al. 2008). In tomato, Pto confers resistance to $P$. syringae pv. tomato DC3000 carrying AvrPto (Martin et al. 1993) in a Pseudomonas resistance and fenthion (Prf)-dependent manner. Transient overexpression of AvrPto in Pto/Prf-transgenic $N$. benthamiana causes a hypersensitive response (HR) (Balmuth and Rathjen 2007; Salmeron et al. 1994; Scofield et al. 1996). However, AvrPto does not affect association between Pto and Prf (Mucyn et al. 2006).

SOBIR1 is generally required for RLP-mediated resistance to bacterial, fungal, and oomycete pathogens (Albert et al. 2015;
Catanzariti et al. 2017; Hegenauer et al. 2016; Jehle et al. 2013; Liebrand et al. 2013; Ma and Borhan 2015; Peng et al. 2015; Takahashi et al. 2016; Zhang et al. 2013a, 2014). Therefore, we hypothesized that this regulatory coreceptor is targeted by cytoplasmic effectors of such pathogens in order to suppress host defense responses. Here, we show that tomato SOBIR1, its homolog SISOBIR1-like, and its orthologs from Arabidopsis and $N$. benthamiana interact with AvrPto, independent of SOBIR1 kinase activity. In addition, AvrPto suppresses AtSOBIR1-induced constitutive immunity in $N$. benthamiana. Furthermore, AvrPto was found to suppress the Cf-4/Avr4-triggered HR, without affecting Cf-4/S1SOBIR1/SISERK3a complex formation.

\section{RESULTS}

\section{AvrPto interacts with SOBIR1 in planta.}

To investigate whether AvrPto and SOBIR1 interact, a binary construct containing C-terminally hemagglutinin (HA) epitopetagged AvrPto was generated. AvrPto-HA was coinfiltrated with Cterminally tagged SISOBIR1-eGFP (enhanced green fluorescent protein) (Liebrand et al. 2013) in N. benthamiana by Agrobacteriummediated transient expression (agroinfiltration). AvrPto-HA was also coinfiltrated with C-terminally tagged SISERK3a-eGFP as a positive control, because AtSERK3 was earlier reported to be targeted by AvrPto (Shan et al. 2008). Coimmunoprecipitation (Co-IP) experiments reveal that AvrPto-HA copurifies with SISOBIR1-GFP upon pull-down of the latter using GFP beads (Fig. 1A). Interaction between SISERK3a and AvrPto was also observed (Fig. 1A). In a reciprocal experiment in which SISOBIR1, SISERK3a, and $\beta$-glucuronidase (GUS) were fused to HA and coinfiltrated with AvrPto-eGFP, a pull-down of SISOBIR1 and SISERK3a using HA beads also resulted in copurification of AvrPto-eGFP (Fig. 1B).

In tomato, the SISOBIR1 homolog SISOBIR1-like shares a high similarity in amino acid sequence with SISOBIR1, and, in $N$. benthamiana, two clear SOBIR1 orthologs are present (Liebrand et al. 2013). In addition, the function of SOBIR1 appears to be highly conserved, as both AtSOBIR1 and SISOBIR1 interact with Cf-4 (Liebrand et al. 2013), and AtSOBIR1 can complement the loss of Avr4-triggered cell death in NbSOBIRl(-like)silenced $C f$-4-transgenic $N$. benthamiana plants (Bi et al. 2016; Liebrand et al. 2013). To study if the SOBIR1 homolog and its orthologs are also targeted by AvrPto, C-terminally eGFP-tagged SISOBIR1-like and NbSOBIR1 were generated and, together with SISOBIR1-eGFP and AtSOBIR1-eGFP (Bi et al. 2016; Liebrand et al. 2013), were coinfiltrated with AvrPto-HA in $N$. benthamiana. Co-IP experiments reveal that, in all cases, AvrPto copurifies with the SOBIR1 variants (Fig. 2).

\section{Kinase activity of SOBIR1 is not required for its interaction with AvrPto.}

SOBIR1 is a so-called "RD" kinase and by its constitutive interaction with RLPs, bipartite RLKs are formed. In this bipartite RLK, SOBIR1 is thought to provide the interacting RLP with a kinase domain to initiate defense signaling upon ligand recognition by the RLP (Gust and Felix 2014; Liebrand et al. 2014). It has been reported that AvrPto targets Pto and FLS2, while the interaction is dependent on kinase activity of these two targets (Xiang et al. 2008; Xing et al. 2007). To determine if kinase activity of SOBIR1 is required for its interaction with AvrPto, eGFP-tagged wild type and kinase-dead SOBIR1 variants (Liebrand et al. 2013) (mutated in the catalytic aspartate [D] of the kinase domain) of Arabidopsis (AtSOBIR1 ${ }^{\mathrm{D} 489 \mathrm{~N}}$ ) and tomato SOBIR1 (SISOBIR1 ${ }^{\text {D473N }}$ ) were coinfiltrated with AvrPtoHA. Co-IP experiments reveal that AvrPto copurifies with all SOBIR1 variants upon their purification using GFP beads, indicating that kinase activity of SOBIR1 is not required for its interaction with AvrPto (Fig. 3). 
Overexpression of $A t S O B I R 1$ induces constitutive immunity, which requires a functional kinase domain.

SOBIR1 is a positive regulator of immunity (Gao et al. 2009), and we anticipated that the protein would constitutively induce an immune response, visualized as cell death, when it accumulates at relatively high levels. To investigate this, eGFPtagged AtSOBIR1, SISOBIR1, SISOBIR1-like, and NbSOBIR1 were transiently overexpressed in leaves of $N$. tabacum by agroinfiltration. Interestingly, only overexpression of AtSOBIRI induced cell death, which was visible within 2 to 3 days after agroinfiltration (Fig. 4A). The other SOBIR1 variants that were tested did not exhibit this constitutive immunity symptom. Although protein accumulation was not tested in N. tabacum, the same constructs were expressed in $N$. benthamiana and resulted in clear protein accumulation for all constructs (discussed below) (Supplementary Fig. S1A). To determine if kinase activity of AtSOBIR 1 is specifically required to induce constitutive immunity, eGFP-tagged AtSOBIR $1^{D 489 N}$ was transiently overexpressed in leaves of $N$. tabacum (Fig. 4B). This kinase-dead SOBIR1 mutant did not induce cell death upon its transient overexpression, indicating that a functional kinase domain is required for AtSOBIR1 to induce constitutive immunity in
$N$. tabacum. This suggests that the constitutive immune response is triggered as a result of perturbation of the immune system of the plant, through constitutive activation of downstream immune signaling components.

The same constructs were tested for constitutive immune activation in $N$. benthamiana. No cell death was observed for any of the SOBIRl variants when expressed alone (data not shown), although all proteins do accumulate. However, when coexpressed with the silencing suppressor P19 (Voinnet et al. 2015), cell death could be observed for AtSOBIR1 at 2 to 3 days after infiltration and, again, not for any of the other tested SOBIR1 variants (Fig. 4A). N. tabacum is generally more sensitive to overexpression of immunity-related proteins and, probably, therefore does not require coexpression of a silencing suppressor to provoke AtSOBIR1 constitutive immunity (Van der Hoorn et al. 2000; Zhang et al. 2013b).

To obtain additional support that this cell-death phenotype indeed reflects an immune response, we analyzed MAPK activation, which is a key downstream step in defense activation (Stulemeijer et al. 2007; Xu et al. 2014). Upon Avr4 recognition by $\mathrm{Cf}-4$ in stable transgenic $N$. benthamiana expressing tomato $C f-4$, immune blots showed a typical MAPK activation pattern
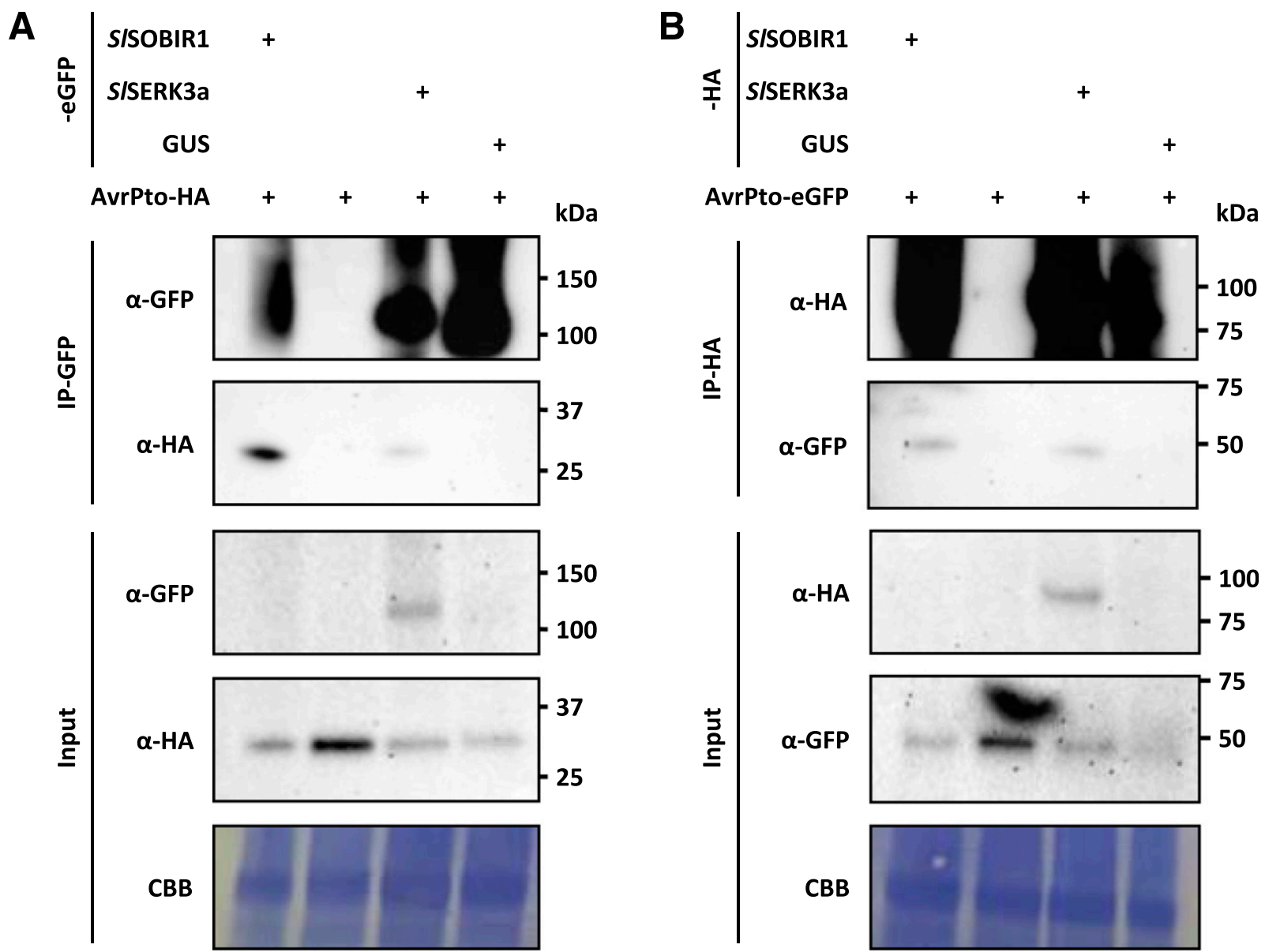

Fig. 1. AvrPto interacts with SISOBIR1 in planta. A, AvrPto-hemagglutinin (HA) interacts with SISOBIR1-eGFP (enhanced green fluorescent protein). Cterminally eGFP-tagged SISOBIR1, SISERK3a, and GUS ( $\beta$-glucuronidase) were coexpressed with AvrPto-HA by agroinfiltration in Nicotiana benthamiana. Two days later, total proteins were extracted and were subjected to immunoprecipitation using GFP_TrapA affinity beads. Total proteins (Input) and immunoprecipitated proteins (IP) were subjected to sodium dodecyl sulfate-polyacrylamide gel electrophoresis (SDS-PAGE) and were blotted to polyvinylidene diflouride (PVDF) membrane. Blots were probed with $\alpha$-GFP antibodies, to detect the immunoprecipitated eGFP-tagged proteins, and with $\alpha$-HA antibodies, to detect coprecipitated AvrPto-HA. $\mathrm{CBB}=$ Coomassie brilliant blue. Experiments were performed at least three times and representative images are shown. B, AvrPto-eGFP interacts with SISOBIR1-HA and SISERK3a-HA. C-terminally HA-tagged SISOBIR1, SISERK3a, and GUS were coexpressed with AvrPto-eGFP in N. benthamiana. Two days later, total proteins were extracted and subjected to immunoprecipitation using HA magnetic beads. Total proteins (Input) and immunoprecipitated proteins (IP) were subjected to SDS-PAGE and were blotted to PVDF membrane. Blots were probed with $\alpha$-HA antibodies to detect the immunoprecipitated HA-tagged proteins and with $\alpha$-GFP antibodies to detect coprecipitated AvrPto-eGFP. Experiments were performed at least three times and representative images are shown. 
(Fig. 4C). Notably, induction of cell death upon AtSOBIRI overexpression in $N$. benthamiana also coincided with MAPK activation (Fig. 4C and D). In conclusion, these data show that the cell death caused by AtSOBIR1 is dependent on its kinase activity and represents a constitutive immune response.

\section{AvrPto suppresses}

AtSOBIR1-induced constitutive immunity.

To determine whether AvrPto can suppress the cell death response induced by overexpression of AtSOBIR1, AvrPtoeGFP and GUS-eGFP were coinfiltrated with AtSOBIR1-eGFP and P19 in N. benthamiana. Coinfiltration of GUS-eGFP does not affect AtSOBIR1-induced cell death, whereas the cell-death response is strongly suppressed by AvrPto-eGFP, indicating that AtSOBIR1-induced constitutive immunity is suppressed by AvrPto (Fig. 5).

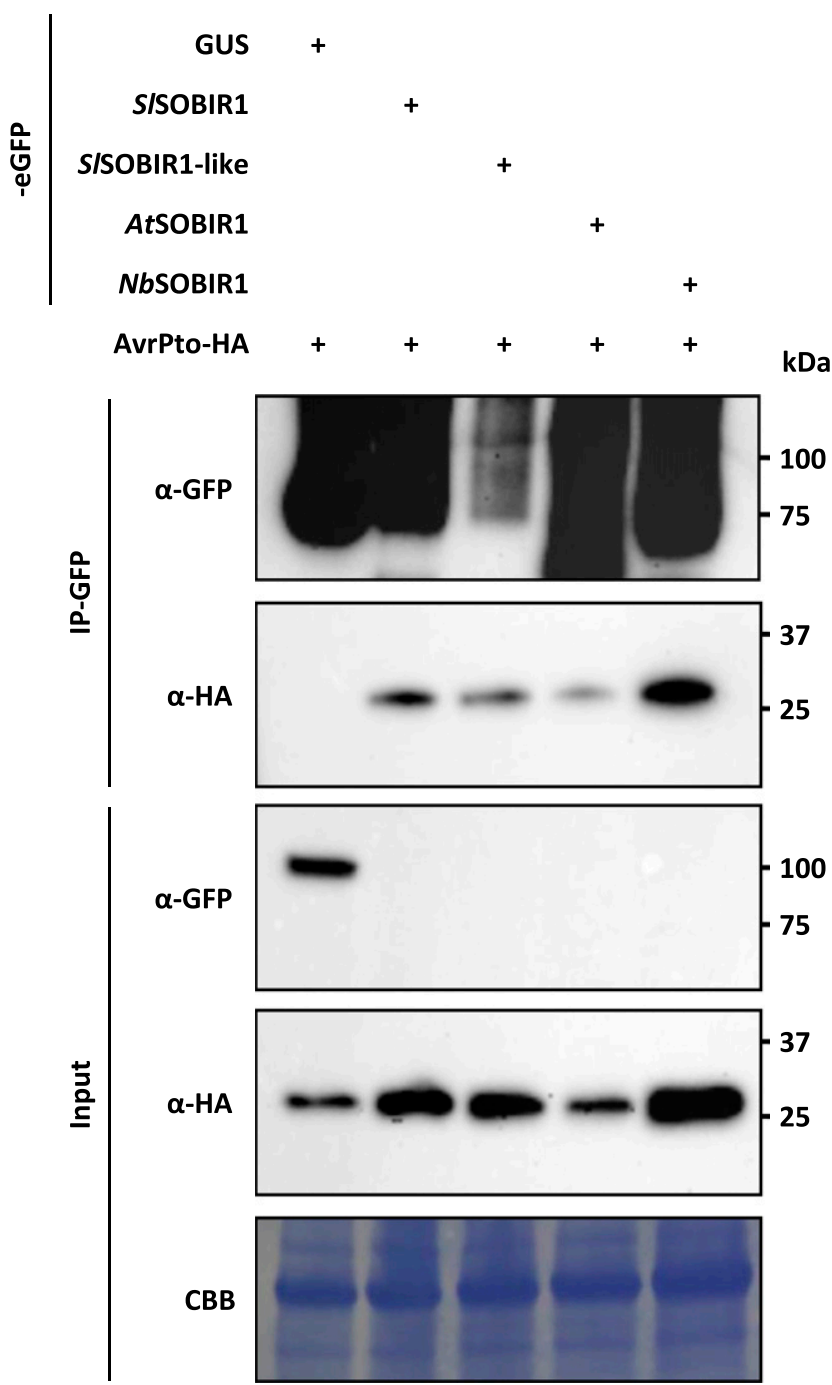

Fig. 2. AvrPto interacts with SISOBIR1, SISOBIR1-like, AtSOBIR1, and NbSOBIR1. C-terminally enhanced green fluorescent protein (eGFP)tagged GUS ( $\beta$-glucuronidase), SlSOBIR1, SlSOBIR1-like, AtSOBIR1, and NbSOBIRl were coexpressed with AvrPto-HA (hemagglutinin) by agroinfiltration in Nicotiana benthamiana. Two days later, total proteins were extracted and subjected to immunoprecipitation using GFP_TrapA affinity beads. Total proteins (Input) and immunoprecipitated proteins (IP) were subjected to sodium dodecyl sulfate-polyacrylamide gel electrophoresis and were blotted to polyvinylidene diflouride membrane. Blots were probed with $\alpha$-GFP antibodies to detect the immunoprecipitated eGFP-tagged proteins and with $\alpha-\mathrm{HA}$ antibodies to detect coprecipitated AvrPto-HA. $\mathrm{CBB}=$ Coomassie brilliant blue. Experiments were performed at least three times and representative images are shown.
AvrPto suppresses the Avr4-triggered cell death in $\boldsymbol{C f}$-4-transgenic $\boldsymbol{N}$. benthamiana plants.

SOBIR1 is required for the Cf-4-mediated cell death upon recognition of Avr4 and for resistance to C. fulvum (Liebrand et al. 2013). Furthermore, kinase activity of SOBIR1 is necessary for $\mathrm{Cf}-4$ function, as a kinase-inactive variant of AtSOBIR1 fails to complement the loss of Avr4-triggered cell death in NbSOBIRI (-like)-silenced Cf-4-transgenic N. benthamiana plants (Bi et al. 2016; Liebrand et al. 2013).

To study if AvrPto is able to suppress the Avr4-triggered cell death through suppression of SOBIR1-mediated defense signaling, AvrPto-eGFP and GUS-eGFP were transiently overexpressed in $C f$-4-transgenic $N$. benthamiana plants, 2 days before agroinfiltration of Avr4. Figure 6 shows that cell death triggered by Avr4 is strongly suppressed upon infiltration of AvrPto-eGFP when compared with infiltration of GUS-eGFP. Together with the observation that AvrPto suppresses AtSOBIR1-induced constitutive immunity (Fig. 5), the suppression of Avr4-triggered cell death by AvrPto indicates that AvrPto compromises Avr4-triggered
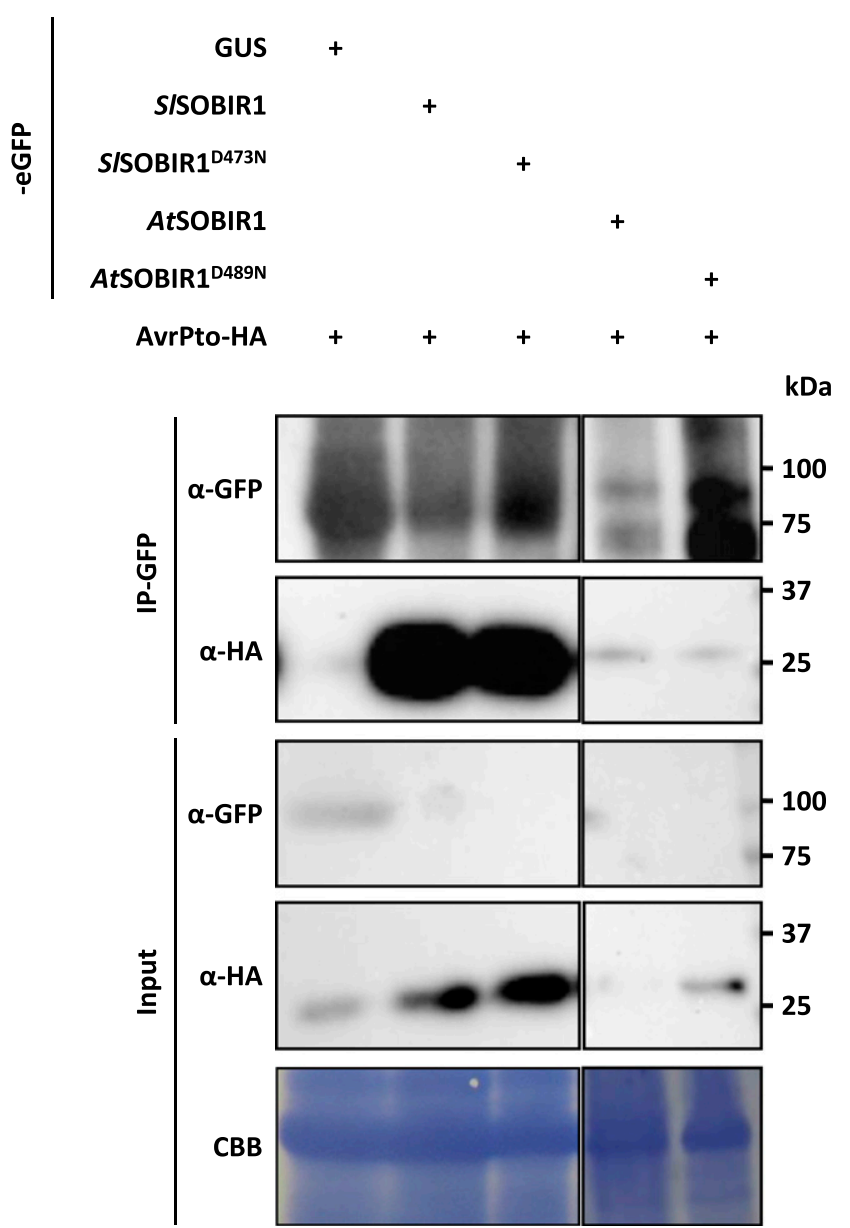

Fig. 3. Kinase activity of SISOBIR1 and AtSOBIR1 is not required for their interaction with AvrPto. C-terminally enhanced green fluorescent protein (eGFP)-tagged GUS ( $\beta$-glucuronidase), SISOBIR1, kinase-dead SISOBIR1 ${ }^{D 473 N}$, AtSOBIRI, and kinase-dead AtSOBIR $1^{D 489 N}$ were coexpressed with AvrPto$H A$ (hemagglutinin) by agroinfiltration in Nicotiana benthamiana. Two days later, total proteins were extracted and subjected to immunoprecipitation, using GFP_TrapA affinity beads. Total proteins (Input) and immunoprecipitated proteins (IP) were subjected to sodium dodecyl sulfatepolyacrylamide gel electrophoresis and were blotted to polyvinylidene diflouride membrane. Blots were probed with $\alpha$-GFP antibodies to detect the immunoprecipitated eGFP-tagged proteins and with $\alpha$-HA antibodies to detect coprecipitated AvrPto-HA. CBB = Coomassie brilliant blue. Experiments were performed at least three times and representative images are shown. 
cell death through suppression of SOBIR1-mediated defense signaling.

\section{AvrPto does not affect \\ Cf-4/SISOBIR1/SISERK3a complex formation.}

Recently it was shown that SISERK3a is involved in the Cf-4 triggered signaling pathway (Postma et al. 2016). SOBIR1 constitutively interacts with Cf-4 (Liebrand et al. 2013), whereas SISERK3a interacts with Cf-4 in an Avr4-dependent manner, as SISERK3a is specifically recruited to the Cf-4/SISOBIR1 complex in the presence of Avr4 (Postma et al. 2016). Silencing of either NbSOBIRI(-like) or $N b S E R K 3 a / b$ compromises the Avr4-triggered cell death (Liebrand et al. 2013; Postma et al. 2016).

To study the effect of AvrPto on Cf-4/SISOBIR1/S1SERK3a complex formation, AvrPto-HA was coinfiltrated with Cf-4-eGFP, SISOBIR1-HA, and SISERK3a-Myc, followed by infiltration of Avr4 protein. Cf-4-eGFP and SISOBIR1-HA are invisible in the input but are readily detectable in the immunoprecipitate (Fig. 7), which is consistent with our earlier published studies (Liebrand et al. 2013; Postma et al. 2016). Co-IP experiments reveal that
AvrPto does not affect the interaction between Cf-4 and SISOBIR1. Moreover, the recruitment of SISERK3a to the Cf-4/SISOBIR1 complex upon infiltration of Avr4 protein is not affected (Fig. 7). These results indicate that AvrPto does not suppress the Avr4triggered cell death by hampering Cf-4/SISOBIR1/SISERK3a complex formation upon Avr4 recognition by Cf-4.

\section{DISCUSSION}

In this study, we provide evidence that AvrPto interacts with AtSOBIR1 and its orthologs of tomato and $N$. benthamiana, independent of the kinase activity of SOBIR1. Overexpression of AtSOBIRl induces constitutive immunity, which is suppressed by AvrPto. In addition, AvrPto also suppresses Cf-4/Avr4-triggered cell death without affecting Cf-4/SISOBIR1/SISERK3a complex formation.

AtSOBIR1 induces a constitutive immune response.

AtSOBIR1 is a functional ortholog of NbSOBIR1 (Liebrand et al. 2013) and, in contrast to NbSOBIR1 or SISOBIR1,

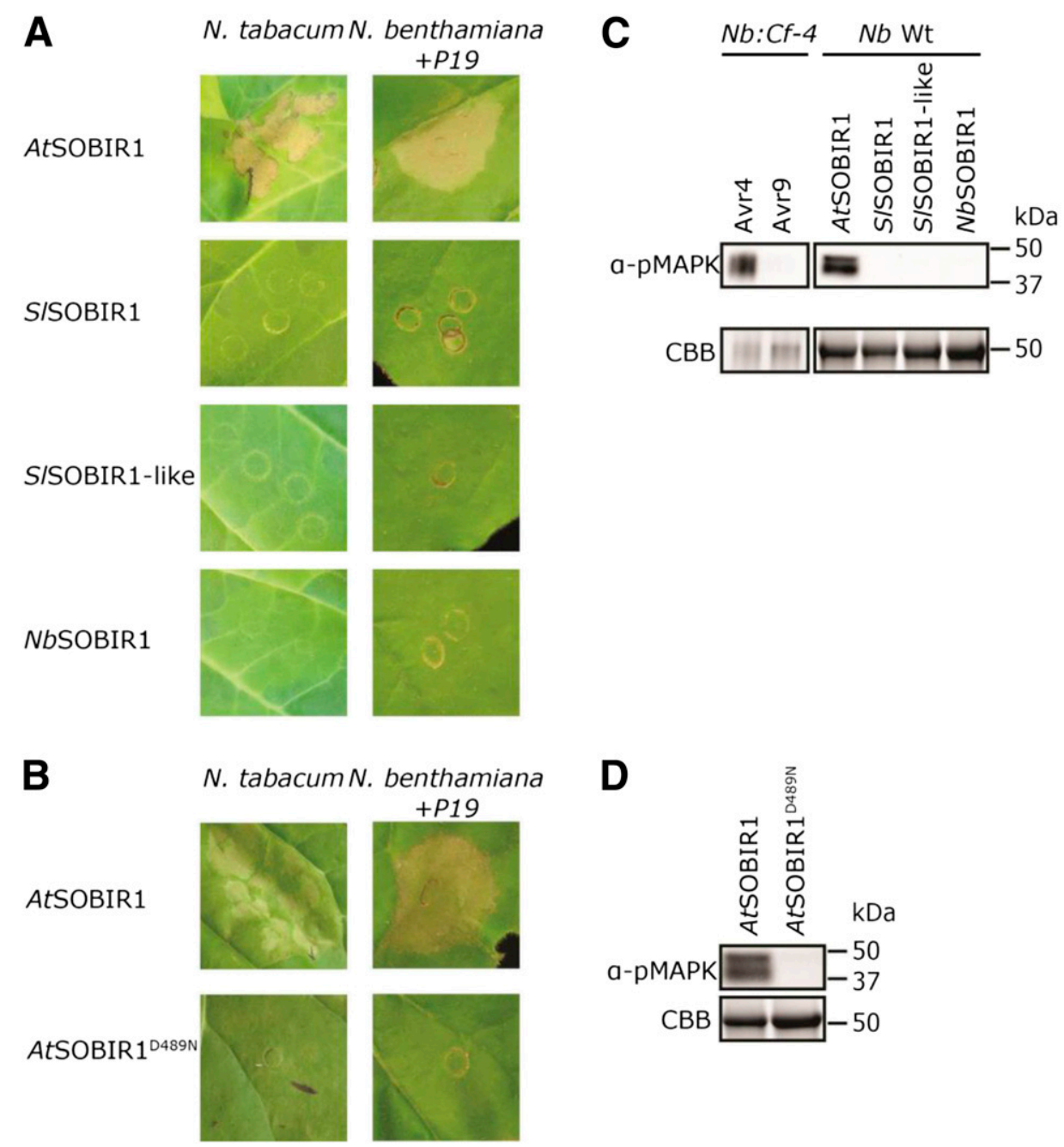

Fig. 4. Constitutive immunity induced by overexpression of AtSOBIR1 is dependent on a functional kinase domain. A, Transient expression of AtSOBIR1 induces cell death in Nicotiana tabacum and N. benthamiana. C-terminally enhanced green fluorescent protein (eGFP)-tagged AtSOBIR1, SISOBIR1, SISOBIRI-like, and NbSOBIRl were transiently expressed by agroinfiltration at an optical density (OD) of 1 ; in $N$. benthamiana, all constructs were coexpressed with the silencing suppressor $P 19$ at an OD of 1 . Pictures were taken at 2 days postinfiltration (dpi) for $N$. tabacum and at 3 dpi for $N$. benthamiana. B, Transient expression of C-terminally eGFP-tagged kinase-dead AtSOBIR ${ }^{D 489 N}$ does not induce cell death in $N$. tabacum or $N$. benthamiana. The experiment was performed as described in A. C, Mitogen-activated protein kinases (MAPKs) are activated upon Avr4 recognition by Cf-4 and upon overexpression of AtSOBIR1. Left, $2 \mu \mathrm{M}$ of Avr4 or Avr9 protein was infiltrated in $C f$-4-transgenic $N$. benthamiana plants, and after $15 \mathrm{~min}$, total protein was extracted and analyzed for MAPK activation, using $\alpha$-p $42 / \mathrm{p} 44$ erk antibody. Right, C-terminally eGFP-tagged AtSOBIR1, SISOBIRI, SISOBIR1-like, and NbSOBIR1 were transiently coexpressed with P19 in N. benthamiana. At $2 \mathrm{dpi}$, total protein was extracted and analyzed for MAPK activation, using $\alpha$-p42/p44-ERK antibody. D, MAPKs are not activated upon overexpression of $A t S O B I R 1^{D 489 N}$. The experiment was performed as described in C. Experiments were performed at least three times and representative images are shown. 
overexpression of AtSOBIRI in N. tabacum or N. benthamiana induces constitutive immunity, which is observed as MAPK activation and cell death (Fig. 4). This observation is in agreement with the cell death and defense gene activation observed upon overexpression of AtSOBIRI in Arabidopsis (Gao et al. 2009). The lack of MAPK activation and cell death upon transient expression of NbSOBIR1 and SISOBIR1 could be an effect of proper SOBIR1 signaling regulation in $N$. tabacum and $N$. benthamiana. The signaling capacity of immune receptors is tightly regulated, as immune homeostasis must be maintained and its deregulation may be lethal (Couto et al. 2016).

A way of regulating immune signaling activity is at the level of phosphorylation of the kinase domain of the signaling components. For instance, the Arabidopsis phosphatases PP2A and PP2C38, associating with SERK3 and BIK1, respectively, were found to negatively regulate the activity of their target kinases (Couto et al. 2016; Segonzac et al. 2014). Potential intrinsic differences between phosphatases, from Arabidopsis and solanaceous plants, involved in negatively regulating SOBIR1 signaling could explain the lack of MAPK activation and absence of cell death upon expression of SISOBIR1 or NbSOBIR1 in contrast to AtSOBIR1 in solanaceous plants. It is likely that solanaceous plants can fine-tune the activity of endogenous versions of SOBIR1 in a more controlled manner than the activity of heterologously expressed AtSOBIR1. Thus, it could be that endogenous phosphatases of $N$. tabacum and $N$. benthamiana can properly negatively regulate solanaceous SOBIR1 immune signaling, whereas these phosphatases might, for example, have lower affinity for the more distantly related ortholog AtSOBIR1. This could result in a higher basal level of phosphorylation of the kinase domain of AtSOBIR1. This higher level of phosphorylation might perturb the immune system of the plant and trigger a constitutive immune response in $N$. tabacum and $N$. benthamiana, which is also

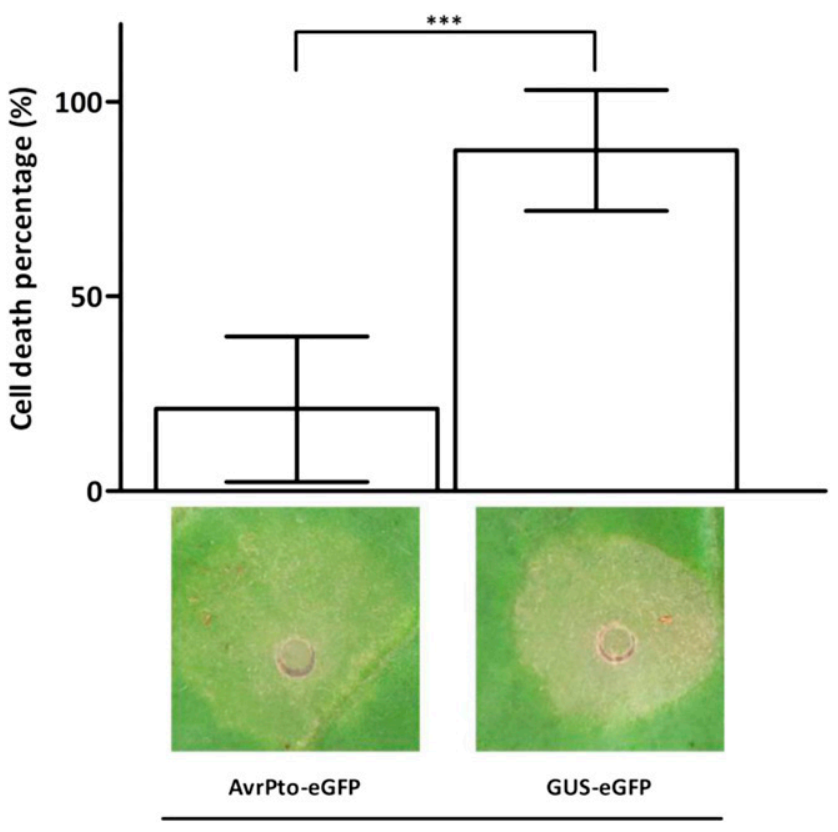

N. benthamiana + AtSOBIR1-eGFP + P19

Fig. 5. Constitutive immunity induced by overexpression of AtSOBIRl is suppressed by AvrPto. AtSOBIR1-eGFP (enhanced green fluorescent protein) and $P 19$ were coexpressed with $\beta$-glucuronidase (GUS)-eGFP or AvrPto$e G F P$ by agroinfiltration in Nicotiana benthamiana at an optical density of 1 . Pictures were taken at 4 days postinfiltration (dpi). Cell death at 4 dpi was scored by its intensity. The means of the cell death percentages are shown. Asterisks indicate a statistically significant difference according to a Student's $t$ test (*** $P<0.0001$ ), with $n=20$. Experiments were performed at least three times and representative images are shown. reflected by constitutive activation of MAPK signaling by AtSOBIR1 (Fig. 4C).

Immunoblot analysis demonstrated that the accumulation levels of the AtSOBIR1 and AtSOBIR $1^{\mathrm{D} 489 \mathrm{~N}}$ proteins inversely correlates with their capacity to activate constitutive immunity. Kinasedead AtSOBIR1 ${ }^{\mathrm{D} 489 \mathrm{~N}}$ accumulates to relatively high levels when compared with wild-type AtSOBIR1 (Fig. 3). Transient expression of wild-type AtSOBIR1 causes constitutive MAPK activation and cell death (Fig. 4), eventually resulting in impaired protein production. AtSOBIR $1^{\text {D489N }}$ lacks constitutive immune activity (Fig. 4) and does not affect the vitality of the cells when transiently expressed, allowing this kinase-dead mutant to accumulate to higher levels than AtSOBIR1 wild type. Although transient expression of SISOBIR1 does not cause MAPK activation and cell death (Fig. 4), its lower accumulation as compared with kinasedead SISOBIR1 ${ }^{\text {D473N }}$ (Fig. 3) suggests that SISOBIR1 also has some residual constitutive immunity activity. This residual activity affects the vitality of the expressing cells and hampers SISOBIR1 accumulation, albeit not as strong as for AtSOBIR1.

\section{AvrPto suppresses Cf-4/SISOBIR1/SISERK3a-triggered cell death without affecting complex formation.}

AvrPto has been reported to suppress cell death triggered by $P$. syringae pv. tomato $\mathrm{T} 1$ in $N$. benthamiana (Kang et al. 2004). In addition, AvrPto has been shown to suppress the flg22/elf26triggered immune response, the latter which is a fragment of the bacterial MAMP ELONGATION FACTOR-TEMPERATURE UNSTABLE (EF-Tu) that is perceived by the RLK EF-Tu RECEPTOR (EFR) (He et al. 2006; Xiang et al. 2008; Zipfel et al. 2006). Here, we show that AvrPto is able to suppress AtSOBIR1-induced constitutive immunity (Fig. 5) as well as the $\mathrm{Cf}-4-$ mediated cell-death response triggered by Avr4

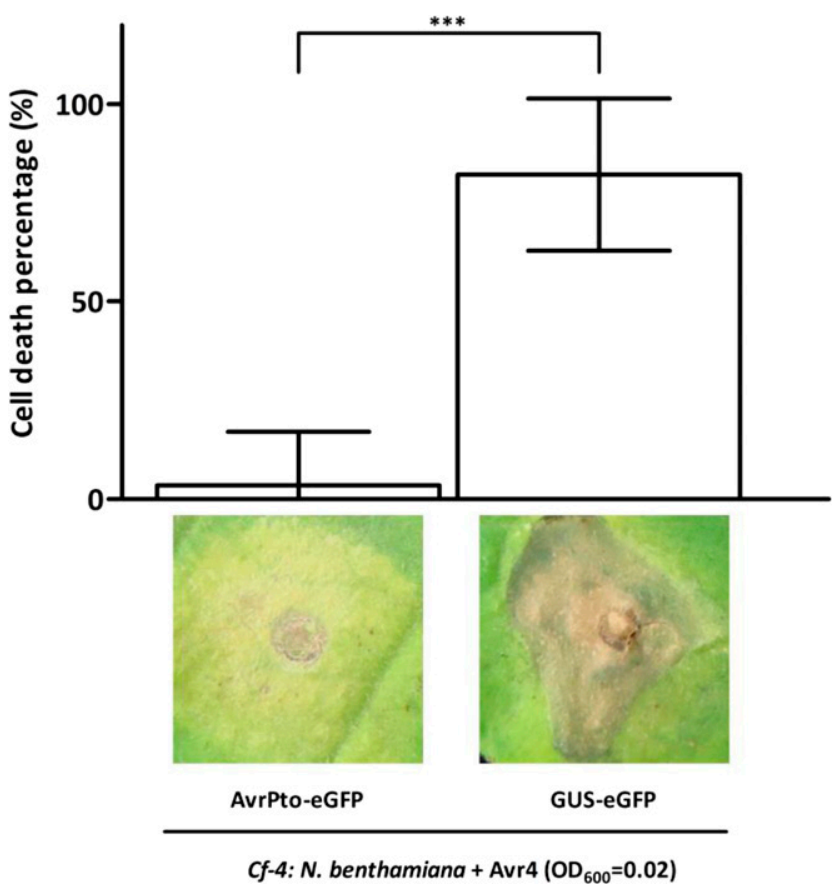

Fig. 6. The Avr4-triggered cell death in Cf-4-transgenic Nicotiana benthamiana plants is suppressed by AvrPto. AvrPto-eGFP (enhanced green fluorescent protein) and $\beta$-glucuronidase (GUS)-eGFP were expressed at an optical density (OD) of 1 in $C f$-4-transgenic $N$. benthamiana plants at 2 days before agroinfiltration of Avr4 at an OD of 0.02. Pictures were taken at 5 days postinfiltration (dpi) of Avr4. Cell death at 5 dpi was scored by the intensity. The means of the cell death percentages are shown. Asterisks indicate a statistically significant difference according to a Student's $t$ test (*** $P<0.0001$ ), with $n=15$. Experiments were performed at least three times and representative images are shown. 
(Fig. 6). Previously, it was reported that AvrPto fails to suppress the cell death triggered by Cf-9/Avr9 (Kang et al. 2004). Cf-9 also interacts with SOBIR1 (Liebrand et al. 2013), and we anticipate that Cf-9 requires SOBIR1 for its function. The absence of AvrPto-mediated suppression of Cf-9/Avr9-induced cell death might be caused by using a lower amount of Agrobacterium expressing AvrPto (optical density at $600 \mathrm{~nm}$ $\left[\mathrm{OD}_{600}\right]=0.4$, as compared with $\mathrm{OD}=1.0$ in our studies). Furthermore, Kang et al. (2004) coinfiltrated AvrPto with Cf9/Avr9, whereas, in our study, AvrPto was infiltrated 2 days before infiltration of Avr4 in Cf-4-transgenic N. benthamiana.

It has been shown that AvrPtoB, which is another T3SS effector of P. syringae pv. tomato DC3000 (Pedley and Martin 2003) and AvrPto both interact with FLS2 and SERK3, whereas only the association of AvrPtoB and FLS2 gets stronger upon flg22 treatment (Göhre et al. 2008; Shan et al. 2008; Xiang et al. 2008). In addition, the flg22-induced FLS2-SERK3 interaction is suppressed by both AvrPtoB and AvrPto (Shan et al. 2008). Furthermore, the dissociation of BIK1 from FLS2 and SERK3 in the presence of flg22 is also suppressed by AvrPto (Lu et al. 2010; Zhang et al. 2010). In view of developmental regulation, SERK3 is involved in the BRASSINOLIDE (BR)triggered signaling pathway, since serk 3 mutants show reduced sensitivity to BR (Li et al. 2002). SERK3 constitutively interacts with the BR receptor BRI1 (Li et al. 2002) and this interaction is also suppressed by AvrPto (Shan et al. 2008).

Altogether, these observations indicate that AvrPto suppresses flg22-triggered plant immunity by interrupting PRR complex formation with the essential coreceptor SERK3 and suppresses BRI1 and SERK3 constitutive complex formation involved in BR-mediated developmental regulation (Shan et al. 2008). In this study, we show that AvrPto is able to suppress Cf4/Avr4-triggered cell death without affecting the constitutive interaction between Cf-4/SISOBIR1 and the Avr4-dependent recruitment of SISERK3a to the Cf-4/SISOBIR1 complex (Figs. 6 and 7). As we found that AvrPto interacts with both SISOBIR1 and SISERK3 (Fig. 1), in this case, AvrPto might interfere in the phosphorylation status of one or both SOBIR1 and SERK3 to suppress Cf-4/Avr4-triggered cell death (Fig. 6).

T3SS effectors interfere in host protein phosphorylation.

Effector-mediated modifications of defense-related proteins have been shown to be an effective way to suppress the immune response (Lee et al. 2013). Interestingly, it has been shown that effectors interfere with the phosphorylation status of important host kinase proteins, including PRRs, RLCKs and MAPKs (Bi and Zhou 2017; Couto and Zipfel 2016; Lee et al. 2013; Macho and Zipfel 2015; Tang et al. 2017). For example, RESISTANCE TO PSEUDOMONAS SYRINGAE PV MACULICOLA 1 (RPM1)INTERACTING PROTEIN 4 (RIN4) associates with and is required for RPM1-mediated resistance (Mackey et al. 2002). The

Fig. 7. Avr4-mediated SISERK3a recruitment to the Cf-4/SISOBIR1 complex is not affected by AvrPto. $C f-4-e G F P$ (enhanced green fluorescent protein), SISOBIRI-HA (hemagglutinin), and SISERK3a-Myc were coexpressed with either $\beta$-glucuronidase (GUS)-HA or AvrPto-HA by agroinfiltration in Nicotiana benthamiana. Two days later, Avr4 or Avr9 protein $(10 \mathrm{nM})$ was infiltrated in the same area, and leaves were harvested 30 min later. Total proteins were extracted and subjected to immunoprecipitation, using GFP_TrapA affinity beads. Total proteins (Input) and immunoprecipitated proteins (IP) were subjected to sodium dodecyl sulfate-polyacrylamide gel electrophoresis and were blotted to polyvinylidene diflouride membrane. Blots were probed with $\alpha$-GFP antibodies to detect immunoprecipitated Cf4-eGFP, with $\alpha$-HA antibodies to detect coprecipitated SISOBIR1-HA, and with $\alpha$-Myc antibodies to detect coprecipitated SISERK3a-Myc. CBB = Coomassie brilliant blue. Experiments were performed at least three times and representative images are shown. The lower $\alpha$-HA panel from the input shows an overexposed blot to reveal accumulation of AvrPto-HA.
P. syringae effector AvrB interacts with RIN4 and induces its phosphorylation, thereby activating the RPM1 signaling pathway leading to resistance to the bacterium (Lee et al. 2015; Mackey et al. 2002). Similar to AvrB, AvrRpm1 also induces RIN4 phosphorylation (Mackey et al. 2002).

Pseudomonas HopAO family members all have tyrosine phosphatase activity, which is required for pathogen virulence (Bretz et al. 2003; Castañeda-Ojeda et al. 2017). For instance,

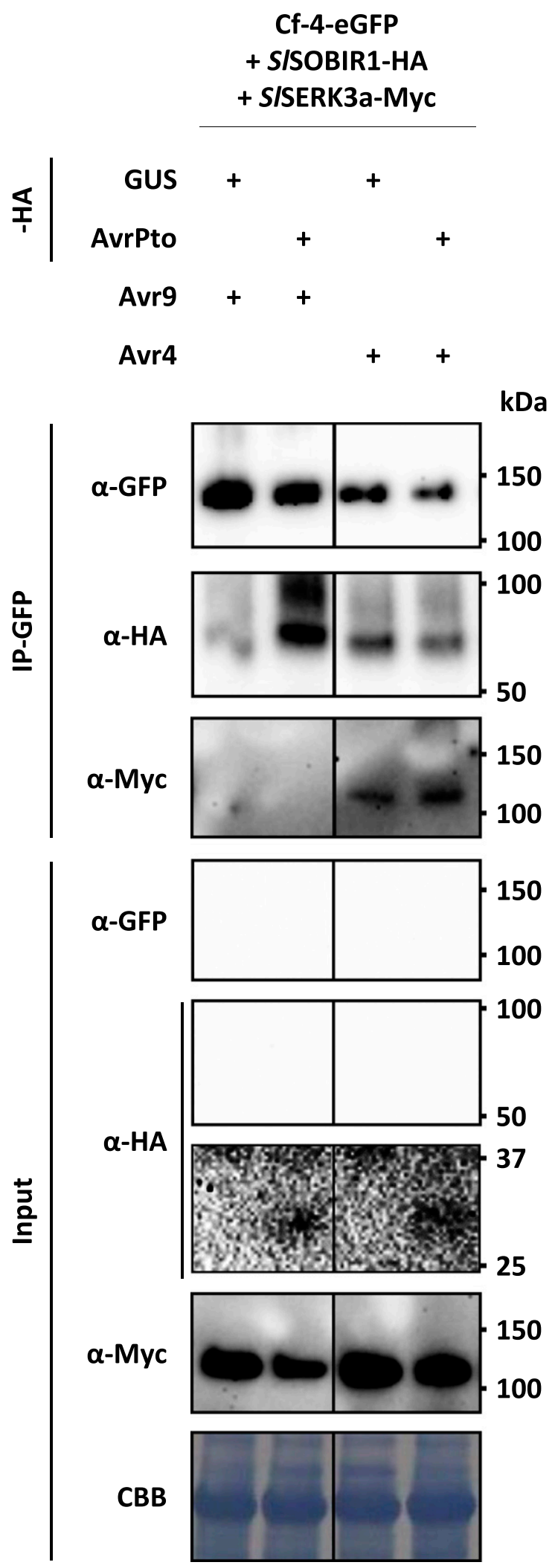

Vol. 31, No. 1, 2018 / 81 
the phosphatase catalytic activity of HopAO1 is required for suppressing callose deposition and MAPK activation upon bacterial colonization of Arabidopsis (Castañeda-Ojeda et al. 2017; Macho et al. 2014; Underwood et al. 2007). Recently, HopAO1 was reported to dephosphorylate EFR on an essential tyrosine residue, a modification occurring upon elf18, another EF-Tu-derived peptide sharing equal activity to elf26 (Zipfel et al. 2006), treatment without interfering in the EFR-SERK3 interaction (Macho et al. 2014). Dephosphorylation of this particular tyrosine residue was found to result in suppression of MTI (Macho et al. 2014).

In another study, it was found that SERK3, which interacts with FLS2 and EFR upon defense activation, is targeted by HopF2, AvrPtoB, and AvrPto (Yasuda et al. 2017). HopF2 targets SERK3 to suppress BIK1 phosphorylation, thereby compromising MAPK activation (Wu et al. 2011; Zhou et al. 2014).

AvrPtoB targets Arabidopsis LysM-RLK CHITIN RECEPTOR KINASE1 (CERK1) to degrade the protein, and this effector also seems to suppress CERK1 kinase activity (Gimenez-Ibanez et al. 2009). In addition, kinase activity of the AvrPtoB tomatointeracting 9 (Bti9) protein, the closest tomato homolog of CERK1, is also suppressed by AvrPtoB (Zeng et al. 2012). AvrPtoB triggers degradation of FLS2/EFR but does not directly affect their kinase activity (Göhre et al. 2008), which contrasts with SERK3, which is not degraded but inhibited in its kinase activity (Cheng et al. 2011).

Cytoplasmic RLCKs, which play a role downstream of PRRs, are phosphorylated by ligand-activated PRRs and, in their turn, transphosphorylate the kinase domain of these PRRs (Couto and Zipfel 2016). Phosphorylation of the RLCK AvrPphB-SUSCEPTIBLE 1 (PBS1) (Swiderski and Innes 2001) is required for its interaction with RESISTANCE TO PSEUDOMONAS SYRINGAE 5 (RPS5) (Warren et al. 1998; Ade et al. 2007). PBS1 is cleaved by HopAR1 (AvrPphB) from $P$. syringae pv. tomato DC 3000 to activate RPS5-mediated auto immunity (Ade et al. 2007; Shao et al. 2003). HopAR1 also dephosphorylates BIK1 to suppress the flg22-induced signaling pathway (Zhang et al. 2010). In addition, BIK1 is also dephosphorylated by the Xanthomonas T3SS effector AvrAC (Feng et al. 2012).

Activation of the MAPK cascade is a common downstream event in plant resistance to pathogens, and the kinase activity of these MAPKs is modified by several bacterial effectors (Lee et al. 2013). HopF2 interacts with MKK5 to prevent its autophosphorylation (Wang et al. 2010), and HopAI1 targets MPK3, MPK6, and MPK4 to dephosphorylate them (Zhang et al. 2007, 2012).

AvrPto has many targets, including SERK3, FLS2, EFR, CERK1, and Pto. Recently, it was reported that both AvrPto overexpression and a double mutation in both SERK3 and $B A K 1$ LIKE1 (BKK1), also referred to as SERK4, similarly suppress oligogalacturonide-induced defense signaling (Gravino et al. 2017). However, it is not clear if AvrPto is able to directly suppress SERK3 kinase activity. AvrPto binds to the kinase domain of FLS2 and EFR, thereby acting as a kinase inhibitor and blocking FLS2 and EFR autophosphorylation to compromise flg22/elf26-triggered immunity (Xiang et al. 2008). Here, we show that AvrPto interacts with SOBIR1; however, it is not known whether AvrPto directly binds to the kinase domain of SOBIR1 or whether this interaction occurs indirectly through another protein that interacts with the SOBIR1 kinase domain. Furthermore, AvrPto targets Pto, thereby suppressing Pto kinase activity (Xing et al. 2007). Surprisingly, the association between BRI1 and SERK3 is interrupted by AvrPto, but the phosphorylation of BRI1 and SERK3 upon BR treatment remains unaffected (Shan et al. 2008).

The overview provided above shows that, except for the BRtriggered phosphorylation of BRI1 and SERK3 that is not affected by AvrPto, the level of phosphorylation of the AvrPtotargeted kinases is lowered by AvrPto. Although there is a debate on the interaction between AvrPto and SERK3 (Xiang et al. 2011), we found that AvrPto targets both SOBIR1 and SERK3 (Fig. 1). Therefore, we anticipate that AvrPto might play a role in altering the phosphorylation status of either SOBIR1, SERK3, or both to suppress Cf-4/Avr4-triggered cell death (Fig. 6), without affecting Cf-4/SISOBIR1/SISERK3a complex formation (Fig. 7). Changing the phosphorylation status of the kinase domain of one or both SOBIR1 and SERK3 could be the result of inhibition of the kinase activity of these RLKs through their targeting by AvrPto.

\section{The requirement of kinase activity of targeted proteins for their interaction with effectors.}

Targeting of host kinases by pathogen effectors is a common strategy to suppress MTI. In most cases, the kinase domain itself is targeted and kinase activity of the targeted proteins plays a role in the interaction. For example, AvrAC interacts with BIK1 to uridylylate the BIK1 phosphorylation site (Feng et al. 2012). Kinase activity of BIK1 is not required for the interaction, but it affects the uridylylation by AvrAC (Feng et al. 2012).

As mentioned above, AvrPtoB targets the kinase domain of SERK3 (Shan et al. 2008), Pto (Kim et al. 2002), FLS2 (Göhre et al. 2008), CERK1 (Gimenez-Ibanez et al. 2009), and Bti9 (Zeng et al. 2012). Kinase activity of SERK3 (Cheng et al. 2011) and Pto (Dong et al. 2009) is required for the interaction with AvrPtoB. However, whether kinase activity is required for interaction with FLS2, CERK1 and Bti9 is unknown.

Kinase activity of Pto is required for its interaction with AvrPto, as it has been shown that the threonine 204 mutant $\mathrm{Pto}^{\mathrm{T} 204 \mathrm{~N}}$, having low kinase activity, only weakly interacts with AvrPto, whereas the kinase-inactive variant Pto ${ }^{\text {S26D }}$ completely loses its interaction with the effector (Xing et al. 2007). Threonine 204 of Pto is highly conserved among the kinase domains of SERK3, SERK4, FLS2, and EFR (Shan et al. 2008). In SERK3, threonine 455 is equivalent to threonine 204 of Pto and a SERK3 $3^{\mathrm{T} 455 \mathrm{~N}}$ mutant has reduced kinase activity (Lin et al. 2014). However, this substitution in SERK3 does not affect its interaction with AvrPto (Shan et al. 2008). It has been shown that Pto has evolved to compete for AvrPto interaction with FLS2 through its ATP-binding site (Xing et al. 2007) and has higher affinity for AvrPto than FLS2 (Xiang et al. 2008). AvrPto targets the ATP-binding site in the kinase domain of FLS2, and a mutation (K898H) in the ATP-binding site of FLS2, which is anticipated to have a lower kinase activity, abolishes its interaction with AvrPto (Xiang et al. 2008; Xing et al. 2007). Although it is unknown whether the kinase domain of SOBIR 1 by itself is sufficient for interaction with AvrPto, kinase activity of SOBIR1 appears not to be required (Fig. 3). This observation reveals different requirements for the interaction of AvrPto with its targets, indicating that AvrPto utilizes different mechanisms to target and affect plant kinases involved in defense signaling.

\section{MATERIALS AND METHODS}

\section{Plant materials and growth conditions.}

Nicotiana benthamiana, Cf-4-transgenic $N$. benthamiana (Gabriëls et al. 2007), and N. tabacum were grown under $16 \mathrm{~h}$ of light at $25^{\circ} \mathrm{C}$ and $8 \mathrm{~h}$ of darkness at $21^{\circ} \mathrm{C}$, in climate chambers, with a relative humidity of $75 \%$.

\section{Binary vectors for agroinfiltrations.}

A fragment consisting of the AvrPto coding sequence in an entry vector was transferred to the destination vector $\mathrm{pBIN}-\mathrm{KS}$ (SOL 2095; for C-terminally tagging with eGFP), to generate 
AvrPto-eGFP, and pGWB14 (SOL 2749; for C-terminally tagging with the HA epitope), to generate AvrPto-HA. SISERK3a-HA, SISOBIR1-HA, SISOBIR1-like-eGFP, SISOBIR $1^{\text {D473N }}$-eGFP, NbSOBIR1-eGFP, GUS-eGFP, and GUS-HA were generated from entry vectors that have been described previously (Bi et al. 2016; Liebrand et al. 2013). SISERK3a-eGFP, SISOBIR1eGFP, SISOBIR1-Myc, Cf-4-eGFP, AtSOBIR1-eGFP, AtSOBIR $1^{\text {D489N }}$-eGFP, and SISERK3a-Myc have been described previously (Bi et al. 2016; Liebrand et al. 2013; Postma et al. 2016). Silencing suppressor P19, which was included in all CoIP experiments, has been described previously (Voinnet et al. 2015).

Binary vectors were transformed to Agrobacterium tumefaciens $\mathrm{C} 58 \mathrm{C} 1$ carrying helper plasmid pCH32. Infiltration of Agrobacterium sp. into plant leaves was performed as described at $\mathrm{OD}_{600}=1$, unless indicated otherwise (Van der Hoorn et al. 2000).

\section{Co-IPs and immunoblotting.}

Co-IPs were performed as described previously (Liebrand et al. 2012). The following antibodies were used: $\alpha$-p42/p44-ERK (New England Biolabs), in combination with goat $\alpha$-rabbit (Sigma) as a secondary antibody, $\alpha$-GFP (Miltenyi Biotec GmbH), $\alpha$-HA (clone 3F10; Roche Applied Science), $\alpha$-cMyc (9E10; Santa Cruz Biotechnology), with sheep $\alpha$-mouse (Amersham) as a secondary antibody.

\section{ACKNOWLEDGMENTS}

We acknowledge T. W. H. Liebrand and R. R. Bye for initiating experiments on AtSOBIR1-induced constitutive cell death in N. tabacum and L. Deurhof for technical support. J. Wu and G. Bi are supported by the China Scholarship Council (CSC). A. M. van der Burgh is supported by the Netherlands Organization for Scientific Research (NWO), division Earth and Life Sciences (ALW)

\section{LITERATURE CITED}

Ade, J., DeYoung, B. J., Golstein, C., and Innes, R. W. 2007. Indirect activation of a plant nucleotide binding site-leucine-rich repeat protein by a bacterial protease. Proc. Natl. Acad. Sci. U.S.A. 104:2531-2536.

Albert, I., Böhm, H., Albert, M., Feiler, C. E., Imkampe, J., Wallmeroth, N., Brancato, C., Raaymakers, T. M., Oome, S., Zhang, H., Krol, E., Grefen, C., Gust, A. A., Chai, J., Hedrich, R., Van den Ackerveken, G., and Nürnberger, T. 2015. An RLP23-SOBIR1-BAK1 complex mediates NLP-triggered immunity. Nat Plants 1:15140-15148.

Asai, T., Tena, G., Plotnikova, J., Willmann, M. R., Chiu, W. L., GomezGomez, L., Boller, T., Ausubel, F. M., and Sheen, J. 2002. MAP kinase signalling cascade in Arabidopsis innate immunity. Nature 415:977-983.

Balmuth, A., and Rathjen, J. P. 2007. Genetic and molecular requirements for function of the Pto/Prf effector recognition complex in tomato and Nicotiana benthamiana. Plant J. 51:978-990.

Bi, G., Liebrand, T. W. H., Bye, R. R., Postma, J., van der Burgh, A. M., Robatzek, S., Xu, X., and Joosten, M. H. A. J. 2016. SOBIR1 requires the GxxxG dimerization motif in its transmembrane domain to form constitutive complexes with receptor-like proteins. Mol. Plant Pathol. 17:96-107.

Bi, G., and Zhou, J. M. 2017. MAP kinase signaling pathways: A hub of plant-microbe interactions. Cell Host Microbe 21:270-273.

Boudsocq, M., Willmann, M. R., McCormack, M., Lee, H., Shan, L., He, P., Bush, J., Cheng, S. H., and Sheen, J. 2010. Differential innate immune signalling via $\mathrm{Ca}^{2+}$ sensor protein kinases. Nature 464:418-422.

Bretz, J. R., Mock, N. M., Charity, J. C., Zeyad, S., Baker, C. J., and Hutcheson, S. W. 2003. A translocated protein tyrosine phosphatase of Pseudomonas syringae pv. tomato DC3000 modulates plant defence response to infection. Mol. Microbiol. 49:389-400.

Castañeda-Ojeda, M. P., Moreno-Pérez, A., Ramos, C., and LópezSolanilla, E. 2017. Suppression of plant immune responses by the Pseudomonas savastanoi pv. savastanoi NCPPB 3335 type III effector tyrosine phosphatases HopAO1 and HopAO2. Front. Plant Sci. 8:680.

Catanzariti, A. M., Do, H. T., Bru, P., de Sain, M., Thatcher, L. F., Rep, M., and Jones, D. A. 2017. The tomato $I$ gene for Fusarium wilt resistance encodes an atypical leucine-rich repeat receptor-like protein whose function is nevertheless dependent on SOBIR1 and SERK3/BAK1. Plant J. 89:1195-1209.

Cheng, W., Munkvold, K. R., Gao, H., Mathieu, J., Schwizer, S., Wang, S., Yan, Y. B., Wang, J., Martin, G. B., and Chai, J. 2011. Structural analysis of Pseudomonas syringae AvrPtoB bound to host BAK1 reveals two similar kinase-interacting domains in a type III Effector. Cell Host Microbe 10:616-626.

Chinchilla, D., Zipfel, C., Robatzek, S., Kemmerling, B., Nürnberger, T., Jones, J. D., Felix, G., and Boller, T. 2007. A flagellin-induced complex of the receptor FLS2 and BAK1 initiates plant defence. Nature 448: 497-500.

Couto, D., Niebergall, R., Liang, X., Bücherl, C. A., Sklenar, J., Macho, A. P., Ntoukakis, V., Derbyshire, P., Altenbach, D., Maclean, D., Robatzek, S., Uhrig, J., Menke, F., Zhou, J. M., and Zipfel, C. 2016. The Arabidopsis protein phosphatase $\mathrm{PP} 2 \mathrm{C} 38$ negatively regulates the central immune kinase BIK1. PLoS Pathog. 12:e1005811.

Couto, D., and Zipfel, C. 2016. Regulation of pattern recognition receptor signalling in plants. Nat. Rev. Immunol. 16:537-552.

Cui, H., Xiang, T., and Zhou, J. M. 2009. Plant immunity: A lesson from pathogenic bacterial effector proteins. Cell. Microbiol. 11:1453-1461.

Dodds, P. N., and Rathjen, J. P. 2010. Plant immunity: Towards an integrated view of plant-pathogen interactions. Nat. Rev. Genet. 11: 539-548.

Dong, J., Xiao, F., Fan, F., Gu, L., Cang, H., Martin, G. B., and Chai, J. 2009. Crystal structure of the complex between Pseudomonas effector AvrPtoB and the tomato Pto kinase reveals both a shared and a unique interface compared with AvrPto-Pto. Plant Cell 21:1846-1859.

Feng, F., Yang, F., Rong, W., Wu, X., Zhang, J., Chen, S., He, C., and Zhou, J. M. 2012. A Xanthomonas uridine 5'-monophosphate transferase inhibits plant immune kinases. Nature 485:114-118.

Feng, F., and Zhou, J. M. 2012. Plant-bacterial pathogen interactions mediated by type III effectors. Curr. Opin. Plant Biol. 15:469-476.

Gabriëls, S. H. E. J., Vossen, J. H., Ekengren, S. K., van Ooijen, G., Abd-ElHaliem, A. M., van den Berg, G. C. M., Rainey, D. Y., Martin, G. B., Takken, F. L. W., de Wit, P. J. G. M., and Joosten, M. H. A. J. 2007. An NB-LRR protein required for HR signalling mediated by both extra- and intracellular resistance proteins. Plant J. 50:14-28.

Gao, M., Wang, X., Wang, D., Xu, F., Ding, X., Zhang, Z., Bi, D., Cheng, Y. T., Chen, S., Li, X., and Zhang, Y. 2009. Regulation of cell death and innate immunity by two receptor-like kinases in Arabidopsis. Cell Host Microbe 6:34-44.

Gimenez-Ibanez, S., Hann, D. R., Ntoukakis, V., Petutschnig, E., Lipka, V., and Rathjen, J. P. 2009. AvrPtoB targets the LysM receptor kinase CERK1 to promote bacterial virulence on plants. Curr. Biol. 19:423-429.

Göhre, V., Spallek, T., Häweker, H., Mersmann, S., Mentzel, T., Boller, T. de Torres, M., Mansfield, J. W., and Robatzek, S. 2008. Plant patternrecognition receptor FLS2 is directed for degradation by the bacterial ubiquitin ligase AvrPtoB. Curr. Biol. 18:1824-1832.

Gómez-Gómez, L., and Boller, T. 2000. FLS2: An LRR receptor-like kinase involved in the perception of the bacterial elicitor flagellin in Arabidopsis. Mol. Cell 5:1003-1011.

Gravino, M., Locci, F., Tundo, S., Cervone, F., Savatin, D. V., and De Lorenzo, G. 2017. Immune responses induced by oligogalacturonides are differentially affected by AvrPto and loss of BAK1/BKK1 and PEPR1/PEPR2. Mol. Plant Pathol. 18:582-595.

Gust, A. A., and Felix, G. 2014. Receptor like proteins associate with SOBIR1-type of adaptors to form bimolecular receptor kinases. Curr. Opin. Plant Biol. 21:104-111.

He, P., Shan, L., Lin, N. C., Martin, G. B., Kemmerling, B., Nürnberger, T., and Sheen, J. 2006. Specific bacterial suppressors of MAMP signaling upstream of MAPKKK in Arabidopsis innate immunity. Cell 125: 563-575.

Hegenauer, V., Fürst, U., Kaiser, B., Smoker, M., Zipfel, C., Felix, G., Stahl, M., and Albert, M. 2016. Detection of the plant parasite Cuscuta reflexa by a tomato cell surface receptor. Science 353:478-481.

Janeway, C. A., Jr. 1989. Approaching the asymptote? Evolution and revolution in immunology. Cold Spring Harb. Symp. Quant. Biol. 54:1-13.

Jehle, A. K., Fürst, U., Lipschis, M., Albert, M., and Felix, G. 2013. Perception of the novel MAMP eMax from different Xanthomonas species requires the Arabidopsis receptor-like protein ReMAX and the receptor kinase SOBIR. Plant Signal. Behav. 8:e27408.

Jones, J. D., and Dangl, J. L. 2006. The plant immune system. Nature 444: 323-329.

Kadota, Y., Shirasu, K., and Zipfel, C. 2015. Regulation of the NADPH oxidase RBOHD during plant immunity. Plant Cell Physiol. 56: $1472-1480$

Kang, L., Tang, X., and Mysore, K. S. 2004. Pseudomonas Type III effector AvrPto suppresses the programmed cell death induced by two nonhost 
pathogens in Nicotiana benthamiana and tomato. Mol. Plant-Microbe Interact. 17:1328-1336.

Kim, Y. J., Lin, N. C., and Martin, G. B. 2002. Two distinct Pseudomonas effector proteins interact with the Pto kinase and activate plant immunity. Cell 109:589-598.

Lee, A. H., Middleton, M. A., Guttman, D. S., and Desveaux, D. 2013. Phytopathogen type III effectors as probes of biological systems. Microb. Biotechnol. 6:230-240.

Lee, D., Bourdais, G., Yu, G., Robatzek, S., and Coaker, G. 2015 Phosphorylation of the plant immune regulator RPM1-INTERACTING PROTEIN4 enhances plant plasma membrane $\mathrm{H}^{+}$-ATPase activity and inhibits flagellin-triggered immune responses in Arabidopsis. Plant Cell 27:2042-2056.

Li, J., Wen, J., Lease, K. A., Doke, J. T., Tax, F. E., and Walker, J. C. 2002 BAK1, an Arabidopsis LRR receptor-like protein kinase, interacts with BRI1 and modulates brassinosteroid signaling. Cell 110:213-222.

Liebrand, T. W. H., Smit, P., Abd-El-Haliem, A., de Jonge, R., Cordewener, J. H. G., America, A. H. P., Sklenar, J., Jones, A. M. E., Robatzek, S., Thomma, B. P. H. J., Tameling, W. I. L., and Joosten, M. H. A. J. 2012 Endoplasmic reticulum-quality control chaperones facilitate the biogenesis of $\mathrm{Cf}$ receptor-like proteins involved in pathogen resistance of tomato. Plant Physiol. 159:1819-1833.

Liebrand, T. W. H., van den Berg, G. C. M., Zhang, Z., Smit, P. Cordewener, J. H. G., America, A. H. P., Sklenar, J., Jones, A. M. E., Tameling, W. I. L., Robatzek, S., Thomma, B. P. H. J., and Joosten, M. H. A. J. 2013. Receptor-like kinase SOBIR1/EVR interacts with receptor-like proteins in plant immunity against fungal infection. Proc. Natl. Acad. Sci. U.S.A. 110:10010-10015.

Liebrand, T. W. H., van den Burg, H. A., and Joosten, M. H. A. J. 2014. Two for all: Receptor-associated kinases SOBIR1 and BAK1. Trends Plant Sci. 19:123-132.

Lin, W., Li, B., Lu, D., Chen, S., Zhu, N., He, P., and Shan, L. 2014. Tyrosine phosphorylation of protein kinase complex BAK1/BIK1 mediates Arabidopsis innate immunity. Proc. Natl. Acad. Sci. U.S.A. 111:3632-3637.

Liu, Y., Huang, X., Li, M., He, P., and Zhang, Y. 2016. Loss-of-function of Arabidopsis receptor-like kinase BIR1 activates cell death and defense responses mediated by BAK1 and SOBIR1. New Phytol. 212:637-645.

Loh, Y. T., and Martin, G. B. 1995. The Pto bacterial resistance gene and the Fen insecticide sensitivity gene encode functional protein kinases with serine/threonine specificity. Plant Physiol. 108:1735-1739.

Lu, D., Wu, S., Gao, X., Zhang, Y., Shan, L., and He, P. 2010. A receptorlike cytoplasmic kinase, BIK1, associates with a flagellin receptor complex to initiate plant innate immunity. Proc. Natl. Acad. Sci. U.S.A. 107:496-501.

Ma, L., and Borhan, M. H. 2015. The receptor-like kinase SOBIR1 interacts with Brassica napus LepR3 and is required for Leptosphaeria maculans AvrLm1-triggered immunity. Front. Plant Sci. 6:933.

Ma, W. 2014. From pathogen recognition to plant immunity: BIK1 cROSses the divide. Cell Host Microbe 15:253-254.

Macho, A. P., Schwessinger, B., Ntoukakis, V., Brutus, A., Segonzac, C., Roy, S., Kadota, Y., Oh, M. H., Sklenar, J., Derbyshire, P., LozanoDurán, R., Malinovsky, F. G., Monaghan, J., Menke, F. L., Huber, S. C., He, S. Y., and Zipfel, C. 2014. A bacterial tyrosine phosphatase inhibits plant pattern recognition receptor activation. Science 343:1509-1512.

Macho, A. P., and Zipfel, C. 2015. Targeting of plant pattern recognition receptor-triggered immunity by bacterial type-III secretion system effectors. Curr. Opin. Microbiol. 23:14-22.

Mackey, D., Holt, B. F., 3rd, Wiig, A., and Dangl, J. L. 2002. RIN4 interacts with Pseudomonas syringae type III effector molecules and is required for RPM1-mediated resistance in Arabidopsis. Cell 108:743-754.

Martin, G. B., Brommonschenkel, S. H., Chunwongse, J., Frary, A., Ganal, M. W., Spivey, R., Wu, T., Earle, E. D., and Tanksley, S. D. 1993. Mapbased cloning of a protein kinase gene conferring disease resistance in tomato. Science 262:1432-1436.

Mucyn, T. S., Clemente, A., Andriotis, V. M., Balmuth, A. L., Oldroyd, G. E., Staskawicz, B. J., and Rathjen, J. P. 2006. The tomato NBARCLRR protein Prf interacts with Pto kinase in vivo to regulate specific plant immunity. Plant Cell 18:2792-2806.

Pedley, K. F., and Martin, G. B. 2003. Molecular basis of Pto-mediated resistance to bacterial speck disease in tomato. Annu. Rev. Phytopathol. 41:215-243.

Peng, K. C., Wang, C. W., Wu, C. H., Huang, C. T., and Liou, R. F. 2015 Tomato SOBIR1/EVR homologs are involved in elicitin perception and plant defense against the oomycete pathogen Phytophthora parasitica. Mol. Plant-Microbe Interact. 28:913-926.

Postma, J., Liebrand, T. W. H., Bi, G., Evrard, A., Bye, R. R., Mbengue, M., Kuhn, H., Joosten, M. H. A. J., and Robatzek, S. 2016. Avr4 promotes
Cf-4 receptor-like protein association with the BAK1/SERK3 receptorlike kinase to initiate receptor endocytosis and plant immunity. New Phytol. 210:627-642.

Salmeron, J. M., Barker, S. J., Carland, F. M., Mehta, A. Y., and Staskawicz, B. J. 1994. Tomato mutants altered in bacterial disease resistance provide evidence for a new locus controlling pathogen recognition. Plant Cell 6 : 511-520.

Scofield, S. R., Tobias, C. M., Rathjen, J. P., Chang, J. H., Lavelle, D. T., Michelmore, R. W., and Staskawicz, B. J. 1996. Molecular basis of genefor-gene specificity in bacterial speck disease of tomato. Science 274: 2063-2065.

Segonzac, C., Macho, A. P., Sanmartín, M., Ntoukakis, V., SánchezSerrano, J. J., and Zipfel, C. 2014. Negative control of BAK1 by protein phosphatase 2A during plant innate immunity. EMBO J. 33:2069-2079.

Shan, L., He, P., Li, J., Heese, A., Peck, S. C., Nürnberger, T., Martin, G. B. and Sheen, J. 2008. Bacterial effectors target the common signaling partner BAK1 to disrupt multiple MAMP receptor-signaling complexes and impede plant immunity. Cell Host Microbe 4:17-27.

Shan, L., Thara, V. K., Martin, G. B., Zhou, J. M., and Tang, X. 2000. The pseudomonas AvrPto protein is differentially recognized by tomato and tobacco and is localized to the plant plasma membrane. Plant Cell 12 2323-2338.

Shao, F., Golstein, C., Ade, J., Stoutemyer, M., Dixon, J. E., and Innes, R. W. 2003. Cleavage of Arabidopsis PBS1 by a bacterial type III effector. Science 301:1230-1233.

Stulemeijer, I. J. E., Stratmann, J. W., and Joosten, M. H. A. J. 2007. Tomato mitogen-activated protein kinases LeMPK1, LeMPK2, and LeMPK3 are activated during the Cf-4/Avr4-induced hypersensitive response and have distinct phosphorylation specificities. Plant Physiol. 144: 1481-1494.

Sun, Y., Li, L., Macho, A. P., Han, Z., Hu, Z., Zipfel, C., Zhou, J. M., and Chai, J. 2013. Structural basis for flg22-induced activation of the Arabidopsis FLS2-BAK1 immune complex. Science 342:624-628.

Swiderski, M. R., and Innes, R. W. 2001. The Arabidopsis PBS1 resistance gene encodes a member of a novel protein kinase subfamily. Plant J. 26 101-112.

Takahashi, T., Shibuya, H., and Ishikawa, A. 2016. SOBIR1 contributes to non-host resistance to Magnaporthe oryzae in Arabidopsis. Biosci. Biotechnol. Biochem. 80:1577-1579.

Tang, D., Wang, G., and Zhou, J. M. 2017. Receptor kinases in plantpathogen interactions: More than pattern recognition. Plant Cell 29: 618-637.

Underwood, W., Zhang, S., and He, S. Y. 2007. The Pseudomonas syringae type III effector tyrosine phosphatase HopAO1 suppresses innate immunity in Arabidopsis thaliana. Plant J. 52:658-672.

Van der Hoorn, R. A., Laurent, F., Roth, R., and De Wit, P. J. 2000 Agroinfiltration is a versatile tool that facilitates comparative analyses of Avr $9 / C f$-9-induced and Avr4/Cf-4-induced necrosis. Mol. Plant-Microbe Interact. 13:439-446.

Voinnet, O., Rivas, S., Mestre, P., and Baulcombe, D. 2015. Retraction: An enhanced transient expression system in plants based on suppression of gene silencing by the p19 protein of tomato bushy stunt virus (Retraction of vol 33, pg 949, 2003). Plant J. 84:846.

Wang, Y., Li, J., Hou, S., Wang, X., Li, Y., Ren, D., Chen, S., Tang, X., and Zhou, J. M. 2010. A Pseudomonas syringae ADP-ribosyltransferase inhibits Arabidopsis mitogen-activated protein kinase kinases. Plant Cell 22:2033-2044.

Warren, R. F., Henk, A., Mowery, P., Holub, E., and Innes, R. W. 1998. A mutation within the leucine-rich repeat domain of the Arabidopsis disease resistance gene RPS5 partially suppresses multiple bacterial and downy mildew resistance genes. Plant Cell 10:1439-1452.

Wu, S., Lu, D., Kabbage, M., Wei, H. L., Swingle, B., Records, A. R., Dickman, M., He, P., and Shan, L. 2011. Bacterial effector HopF2 suppresses arabidopsis innate immunity at the plasma membrane. Mol. Plant-Microbe Interact. 24:585-593.

Xiang, T., Zong, N., Zhang, J., Chen, J., Chen, M., and Zhou, J. M. 2011. BAK1 is not a target of the Pseudomonas syringae effector AvrPto. Mol Plant-Microbe Interact. 24:100-107.

Xiang, T., Zong, N., Zou, Y., Wu, Y., Zhang, J., Xing, W., Li, Y., Tang, X., Zhu, L., Chai, J., and Zhou, J. M. 2008. Pseudomonas syringae effector AvrPto blocks innate immunity by targeting receptor kinases. Curr. Biol. 18:74-80.

Xing, W., Zou, Y., Liu, Q., Liu, J., Luo, X., Huang, Q., Chen, S., Zhu, L., Bi, R., Hao, Q., Wu, J. W., Zhou, J. M., and Chai, J. 2007. The structural basis for activation of plant immunity by bacterial effector protein AvrPto. Nature 449:243-247.

Xu, J., Xie, J., Yan, C., Zou, X., Ren, D., and Zhang, S. 2014. A chemica genetic approach demonstrates that MPK3/MPK6 activation and 
NADPH oxidase-mediated oxidative burst are two independent signaling events in plant immunity. Plant J. 77:222-234.

Yasuda, S., Okada, K., and Saijo, Y. 2017. A look at plant immunity through the window of the multitasking coreceptor BAK1. Curr. Opin. Plant Biol. 38:10-18.

Zeng, L., Velásquez, A. C., Munkvold, K. R., Zhang, J., and Martin, G. B. 2012. A tomato LysM receptor-like kinase promotes immunity and its kinase activity is inhibited by AvrPtoB. Plant J. 69:92-103.

Zhang, J., Li, W., Xiang, T., Liu, Z., Laluk, K., Ding, X., Zou, Y., Gao, M. Zhang, X., Chen, S., Mengiste, T., Zhang, Y., and Zhou, J. M. 2010. Receptor-like cytoplasmic kinases integrate signaling from multiple plant immune receptors and are targeted by a Pseudomonas syringae effector. Cell Host Microbe 7:290-301.

Zhang, J., Shao, F., Li, Y., Cui, H., Chen, L., Li, H., Zou, Y., Long, C., Lan, L., Chai, J., Chen, S., Tang, X., and Zhou, J. M. 2007. A Pseudomonas syringae effector inactivates MAPKs to suppress PAMP-induced immunity in plants. Cell Host Microbe 1:175-185.

Zhang, L., Kars, I., Essenstam, B., Liebrand, T. W. H., Wagemakers, L., Elberse, J., Tagkalaki, P., Tjoitang, D., van den Ackerveken, G., and van Kan, J. A. L. 2014. Fungal endopolygalacturonases are recognized as microbeassociated molecular patterns by the arabidopsis receptor-like protein RESPONSIVENESS TO BOTRYTIS POLYGALACTURONASES1. Plant Physiol. 164:352-364.
Zhang, W., Fraiture, M., Kolb, D., Löffelhardt, B., Desaki, Y., Boutrot, F. F. G., Tör, M., Zipfel, C., Gust, A. A., and Brunner, F. 2013a Arabidopsis receptor-like protein 30 and receptor-like kinase suppressor of BIR1-1/EVERSHED mediate innate immunity to necrotrophic fungi. Plant Cell 25:4227-4241.

Zhang, Z., Fradin, E., de Jonge, R., van Esse, H. P., Smit, P., Liu, C. M., and Thomma, B. P. H. J. 2013b. Optimized agroinfiltration and virus-induced gene silencing to study Ve1-mediated Verticillium resistance in tobacco. Mol. Plant-Microbe Interact. 26:182-190.

Zhang, Z., Wu, Y., Gao, M., Zhang, J., Kong, Q., Liu, Y., Ba, H., Zhou, J., and Zhang, Y. 2012. Disruption of PAMP-induced MAP kinase cascade by a Pseudomonas syringae effector activates plant immunity mediated by the NB-LRR protein SUMM2. Cell Host Microbe 11:253-263.

Zhou, J., Wu, S., Chen, X., Liu, C., Sheen, J., Shan, L., and He, P. 2014. The Pseudomonas syringae effector HopF2 suppresses Arabidopsis immunity by targeting BAK1. Plant J. 77:235-245.

Zipfel, C. 2014. Plant pattern-recognition receptors. Trends Immunol. 35: 345-351.

Zipfel, C., Kunze, G., Chinchilla, D., Caniard, A., Jones, J. D., Boller, T., and Felix, G. 2006. Perception of the bacterial PAMP EF-Tu by the receptor EFR restricts Agrobacterium-mediated transformation. Cell 125:749-760 